\title{
Calcium regulation of nucleocytoplasmic transport
}

\author{
Ashapurna Sarma, Weidong Yang ${ }^{凶}$ \\ Department of Biological Sciences, Center for Photochemical Sciences, Bowling Green State University, Bowling Green, \\ $\mathrm{OH}$ 43403, USA \\ $\triangle$ Correspondence: wyang@bgsu.edu \\ Received March 15, 2011 Accepted March 27, 2011
}

\begin{abstract}
Bidirectional trafficking of macromolecules between the cytoplasm and the nucleus is mediated by the nuclear pore complexes (NPCs) embedded in the nuclear envelope (NE) of eukaryotic cell. The NPC functions as the sole pathway to allow for the passive diffusion of small molecules and the facilitated translocation of larger molecules. Evidence shows that these two transport modes and the conformation of NPC can be regulated by calcium stored in the lumen of nuclear envelope and endoplasmic reticulum. However, the mechanism of calcium regulation remains poorly understood. In this review, we integrate data on the observations of calciumregulated structure and function of the NPC over the past years. Furthermore, we highlight challenges in the measurements of dynamic conformational changes and transient transport kinetics in the NPC. Finally, an innovative imaging approach, single-molecule superresolution fluorescence microscopy, is introduced and expected to provide more insights into the mechanism of calcium-regulated nucleocytoplasmic transport.
\end{abstract}

KEYWORDS nuclear envelope, nuclear pore complex, nucleocytoplasmic transport, calcium stores, single-molecule fluorescence microscopy

\section{INTRODUCTION}

A eukaryotic cell is compartmentalized into cytoplasm and nucleus by a double membrane nuclear envelope (NE). Proteins synthesized in the cytoplasm are imported into the nucleus and genetic materials transcribed in the nucleus are exported to the cytoplasm. This bidirectional trafficking of macromolecules across the NE, termed as nucleocytoplasmic transport, is enabled by thousands of nuclear pore complexes (NPCs) embedded in the NE. The NPC is a large assembly composed of approximately 30 different proteins, known as nucleoporins (Nups), with each present in an integer multiple of eight copies (Rout and Blobel, 1993; Rout et al., 2000; Rout and Aitchison, 2001; Cronshaw et al., 2002; Fahrenkrog and Aebi, 2003; Fried and Kutay, 2003; Rout et al., 2003; Suntharalingam and Wente, 2003; Weis, 2003; Beck et al., 2004). Approximately one third of the total Nups possess 'natively unfolded' structure with domains that are rich in phenylalanine-glycine (FG) repeats (Rout and Wente, 1994; Denning et al., 2003; Strawn et al., 2004; Patel et al., 2007). These FG-Nups form the selective permeability barrier in the NPC that allows for two transport modes: (1) passive diffusion of small molecules ( $<20-40 \mathrm{kDa}$ ) and (2) transport receptorfacilitated transport of larger molecules (up to $50 \mathrm{MDa}$ ) (Corbett and Silver, 1997; Kau et al., 2004; Miao and Schulten, 2009).

Transport of macromolecules across the NE is a constitutive process. Alternations in gene expression of the components in NPC or impairments in the factors involved in transport machinery can impede the functional nucleocytoplasmic transport. Additionally, disturbance in the cellular environment like intracellular ionic shifts will also hinder the nucleocytoplasmic transport, which in turn can influence gene expression, signal transduction, and cell development as a whole. In fact, the divalent calcium cation, a major signaling molecule, regulates numerous cell functions and influences almost every aspect of cellular life, starting from fertilization to cell death. Recently, many evidences have indicated that both passive and facilitated nucleocytoplasmic transport modes can be regulated by calcium ions stores in the perinuclear spaces of NE and the cisternal spaces of endoplasmic reticulum (ER). Furthermore, the malfunctioned nucleocytoplasmic transport caused by alternated ionic calcium was 
closely associated to cardiac and neurodegenerative diseases (Kass and Orrenius, 1999; Frey et al., 2000; Mattson et al., 2000; Rizzuto and Pozzan, 2003). With such critical significance in human health, however, the fundamental mechanism of calcium-regulated NPC conformational change and the related functional nucleocytoplasmic transport are still poorly understood. Therefore, it becomes essential to investigate the mechanism of calcium-mediated structural and functional changes in NPC, devise a new method to bridge the gap between our understandings, and provide a clear perception on this topic. In this review, we compiled the studies done so far on calcium-regulated nucleocytoplasmic transport. The discrepancies involved in these investigations were also highlighted. Then, we reviewed on a novel approach, single-molecule fluorescence microscopy, which has been successfully applied in the studies of nucleocytoplasmic transport. Finally, we expect the new method could be expanded to the calcium-mediated structural and functional studies of NPC and provide new insights in this field.

\section{THE NUCLEAR PORE COMPLEX}

Electron microscopy methods revealed that the NPC is one of the largest molecular machines in eukaryotic cells. The central framework encircles the central pore in the NPC with $\sim 40-90 \mathrm{~nm}$ in length and a minimum internal diameter of around $40-75 \mathrm{~nm}$. On the cytoplasmic side of the NPC, the cytoplasmic ring moiety caps the framework with eight short cytoplasmic filaments protruding into the cytoplasm for $\sim 50 \mathrm{~nm}$. On the nuclear side of the NPC, the central framework is capped by a nuclear ring moiety connected with a distal ring by an assembly of eight filaments, forming a nuclear basket extending into the nucleoplasm for $\sim 75 \mathrm{~nm}$ from the inner nuclear membrane (Rout and Blobel, 1993; Fahrenkrog and Aebi, 2003; Lim et al., 2008; Peters, 2009). NPCs are porous to small molecules and ions, while large molecules are repelled unless they are assisted by transport receptors requiring an input of metabolic energy to facilitate the translocation through the NPC. The proteins and macromolecules involved in transport are termed as cargoes, and have short signal sequences based on its transport directionality. The sequences can be either nuclear localizing signal (NLS) for nuclear import or nuclear export signal (NES) for nuclear export. The cargoes can be specifically recognized by soluble accessory proteins (importins/exportins) that mediate the transport of cargo complexes by transient interactions with the FG repeats in the NPC (Rexach and Blobel, 1995; Wozniak et al., 1998; Cook et al., 2007; Stewart, 2007). Importin $\beta$ (Imp $\beta$ )-type transport receptors account for the majority of the nuclear transport pathways (Bayliss et al., 2000). They circulate between nucleus and cytoplasm, recognize cargo molecules and transfer them from one side of the NE to the other (Lyman et al., 2002). Substrate loading to and release from Imp $\beta$-type transport receptors is regulated by a concentration gradient of RanGTP across the NE, which is sensed through the RanGTP binding domains present in the transport receptors (Coutavas et al., 1993; Moore and Blobel, 1993; Bischoff et al., 1994; Yokoyama et al., 1995; Bischoff and Görlich, 1997; Izaurralde et al., 1997; Walther et al., 2002). Multiple studies have shown that a series of rapid and low-affinity binding events between the transport receptors and the FG repeats along the NPC enhance efficient transport of receptor-cargo complexes through the NPC (Bednenko et al., 2003; Isgro and Schulten, 2005; Lee et al., 2005; Liu and Stewart, 2005). RanGTP is only hydrolyzed when the cargo complexes arrive at the cytoplasm after the facilitated diffusion through the NPC (Brohawn et al., 2009).

\section{THE CALCIUM STORES}

The lumen of the ER, holding calcium in millimolar ranges, is coupled to the cisternal spaces between the two bilayers of $\mathrm{NE}$, called the perinuclear space (Erickson et al., 2006). This space acts as the major calcium storage in the eukaryotic cell. The nucleus is surrounded by calcium storage compartment, which sequesters and releases calcium in response to intracellular second messengers. Calcium is an important element in many cellular signal-transduction cascades that modulate gene transcription. Changes in cellular concentration of calcium clearly affect many cellular functions. Studies have shown that the calcium stores are regulated by calcium channels located on both the cytoplasmic and the nucleoplasmic faces of the NE. Inositol (1,4,5)-trisphosphate receptors $\left(\mathrm{InsP}_{3} \mathrm{Rs}\right)$ is one of them which are present on both sides of NE. Binding of inositol 1,4,5-trisphosphate to the channel opens the channels and releases calcium from the stores into the cytoplasm or the nucleus. Other receptors involved in calcium signaling are the rynodine receptors and nicotinic acid adenine phosphate receptors (Kramer et al., 2007; Bootman et al., 2009). On the contrary, to maintain the high calcium concentrations in the calcium stores, ATPdependent calcium uptake pumps sequester calcium back into the stores (Carmen et al., 1997; Malviya and Klein, 2006). However, if calcium pumps mechanism is disabled, for example, by thapsigargin $(\mathrm{Tg})$, a specific inhibitor of the calcium ATPase, the calcium storage can be reduced. Other chemicals like ethylene glycol tetraacetic acid (EGTA), bisaminophenoxy ethane-tetraacetic acid (BAPTA), which are rapid calcium ion chelators, can also be used. Calcium concentration can be depleted by a specific calcium ion ionophore like ionomycin or A23187 (Gerasimenko et al., 2003; Lim et al., 2007) as well. Most of the studies discussed in this review have used these chemicals to reduce the calcium concentration from the stores.

Numerous studies in past two decades have proposed that NPC structure and the associated functions are regulated by 
changes in calcium concentration either within the lumen of the NE, or at the cytosolic-nucleoplasmic face (Carmen et al., 1997; Bootman et al., 2009). However, there is a lot of incongruity in the observed effects both in terms of NPC structural changes and nucleocytoplasmic transport.

\section{CALCIUM-REGULATED STRUCTURAL CHANGES IN NPC}

Depletion of calcium from the stores in the lumen of NE and ER leads to structural changes in NPC. Broadly categorized, the structural changes of NPC were observed on the cytoplasmic side, or on the nuclear side of NPC, or on both sides of the NPC. The main research tools in these studies are atomic force microscopy (AFM) and scanning electron microscopy (SEM), which were employed to scan the threedimensional topography of the NPC structure.

\section{Conformational changes observed from the cytoplasmic side of NPC}

The nucleocytoplasmic transport of intermediate-sized $(20-40 \mathrm{kDa})$ molecules was inhibited by depletion of nuclear cisternal calcium (Stehno-Bittel et al., 1995b), and PerezTerzic et al. associated this result with the structural changes in NPC. Their initial studies with filed-emission SEM and AFM showed two distinct conformational states of NPC with presence or absence of nuclear cisternal calcium on fixed NEs of Xenopus laevis oocyte (Perez-Terzic et al., 1996). The central channel in the NPC was occluded when the nucleus was treated with calcium depleting agents, so they proposed the presence of a central 'plug' which blocks the channel of NPC in response to decreased calcium ion concentration in the lumen of the NE. The AFM images showed both an upward shift of the plug to a blocking position and a change in the internal pore diameter (Fig. 1Aa). There were qualms about observations on the fixed NE of Xenopus laevis oocyte, so the same group came up with new preparation techniques and imaged the NE under more physiological conditions (Wang and Clapham, 1999). They found a $10 \mathrm{~nm}$ shift of the central plug towards the cytoplasmic side and a $30 \%$ decline in the internal pore diameter when stored calcium was depleted (Fig. 1Ab). Also the central plug can be recovered to original position after the stores are replenished with calcium.

A few other research groups also reported the similar calcium-dependent changes in the shape of NPCs. But, they further found that not only calcium but also ATP can induce changes in the NPC structure (Rakowska et al., 1998). Contraction of the pore was also observed in ATP-induced NEs (Danker and Oberleithner, 2000). ATP is believed to be related to calcium-mediated changes, as addition of ATP replenishes the calcium stores and unplugs the central pores in the cytoplasmic side (Perez-Terzic et al., 1996; Wang and Clapham, 1999). While an alternative explanation was proposed that ATP addition enhances the cargo complexes transit through the pore and thus an "unpluged" appearance was captured (Stoffler et al., 1999). Ensslin and his group used AFM to study distinct NPC topologies of xenopus oocyte by different agents and reported a similar change in topology with the depletion of calcium concentrations. They found a plug-like appearance on the cytoplasmic side of the oocyte NPC (Jäggi et al., 2003), as shown in Fig. 1Ac. Apart from the usage of amphibian NPC in the studies, a few other research groups used mammalian cells like cardiac cells from rats to study the effect of calcium, and found that the cardiac NPCs also displayed an apparent closer of the nuclear pore with a plug-like appearance (Perez-Terzic et al., 1999), as shown in Fig. 1 Ad.

Many argue that the central plug/mass observed in the calcium-related structural studies of NPC might be a cargo caught in transit, or an integral component of NPC participating in transport, or even a collapsed nuclear basket (Stoffler et al., 2006). The cryoelectron tomography of fully native NPCs from the Xenopus oocyte NE revealed a solid but feebly structured mass, with multiple configurations, in the center of the pore. It was found that the pores remained unplugged under optimal transport state, while the pores appeared to be plugged in transport inhibited state (at a temperature of $4^{\circ} \mathrm{C}$ when the samples were prepared) (Stoffler et al., 2003). Thus, a conclusion was drawn that the calcium-induced central plug is a cargo caught in transit. Another explanation is that the distal ring on the nuclear side of NPC was induced to move towards the central pore and occlude the nuclear entry of the central pore which gives a plug-like appearance (Wang and Clapham, 1999). In addition, FG repeats present in the NPC tethered to the cytoplasmic and nuclear periphery are mobile and extended, which could interact with each other to form a 'transient plug' in the center (Paulillo et al., 2006).

\section{Conformational changes observed from the nuclear side of NPC}

On the contrary, Aebi et al. did not find any difference in the cytoplasmic face; instead, they found that the morphology of the nuclear face of NPC has changed significantly (Stoffler et al., 1999). They employed time lapse AFM to monitor the calcium-mediated structural changes of NPC from both the cytoplasmic and the nuclear sides. A modified isolation/ fixation preparation protocol of Xenopus laevis, which avoids chemical fixation and harsh dehydration/rehydration, was developed to reproduce a 'visually native NPC under native conditions' by this group. The AFM imaging revealed asymmetric NPC appearance on both sides-the 'dome like' nuclear side and the 'donut like' cytoplasmic side. They did not find any significant structural changes in the cytoplasmic face (Fig. 1Ba), but two distinct morphological states on the nuclear side in response to calcium concentrations in the 
A

a

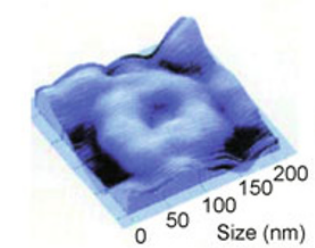

b

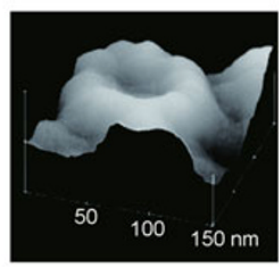

c
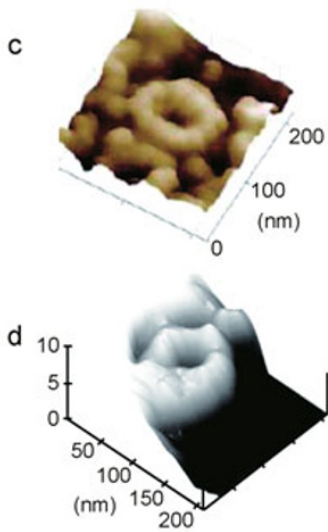

- Calcium
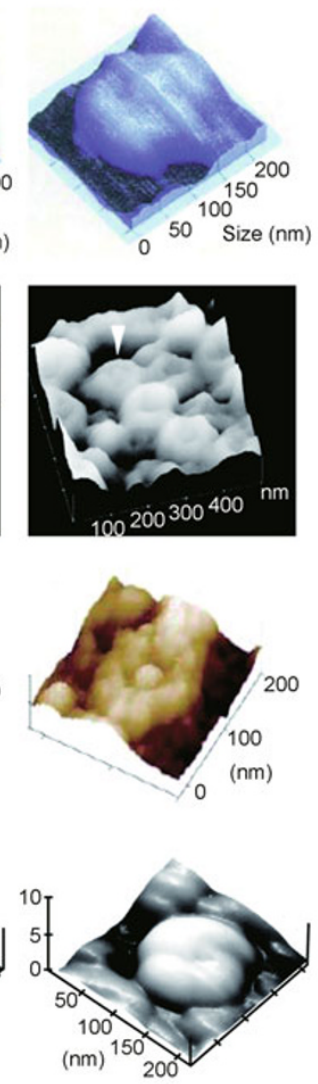

B

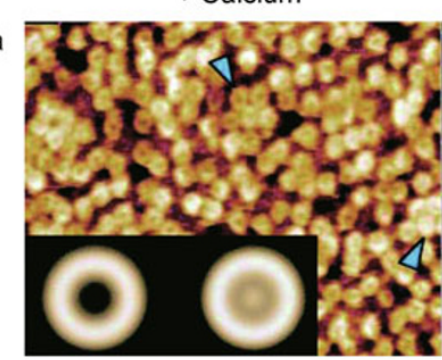

Cytoplasmic side of NPC

b

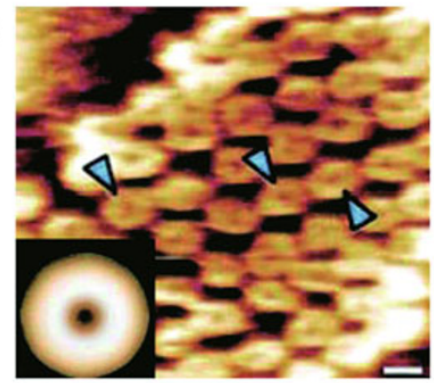

Nuclear side of NPC
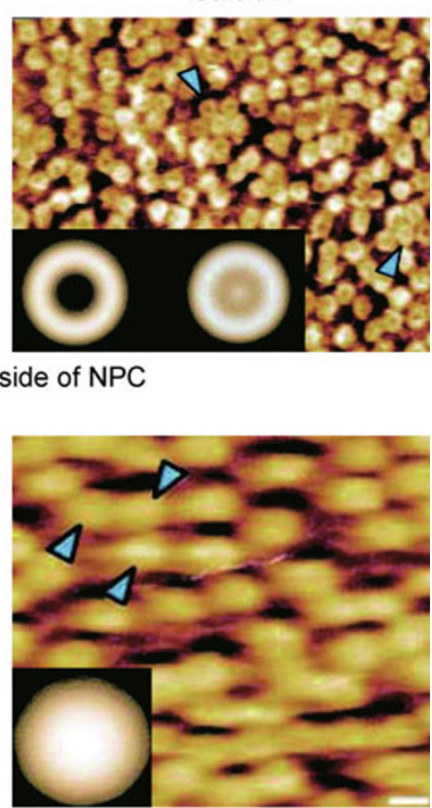

Cytoplasmic side of NPC

Figure 1. Structural changes in NPC with luminal calcium depletion. (A) Calcium-induced structural changes observed in the cytoplasmic side of nuclear pore complex (NPC) in different types of cells. a and b. Images from fixed Xenopus oocyte nuclear envelopes detected by atomic force topography show the open and closed states in the axial central region of NPC in the presence (+ Calcium) and absence (- Calcium) of calcium. Figure adopted from the references of (Perez-Terzic et al., 1996; Wang and Clapham, 1999). c. Topologies of fixed Xenopus oocyte nuclear envelopes by scanning electron microscopy (SEM) also show the open and closed states in the presence and absence of calcium. Figure adopted from (Jäggi et al., 2003). d. Atomic-force topographic images from fixed NPC of cardiomyocytes demonstrate the open and closed states of NPC in the presence and absence of calcium. Figure adopted from (Perez-Terzic et al., 1999). (B) Images show structural changes in native Xenopus oocyte NPC's by atomic force microscopy (AFM) in contact mode. a. Atomic-force topographic images of native Xenopus oocyte NPCs show no significant structural change in the cytoplasmic face in response to calcium changes. b. Images from native Xenopus oocyte NPCs indicate the calcium-mediated opening and closing of the nuclear baskets by time-lapse AFM. Figures adopted from (Stoffler et al., 1999).

stores (Fig. 1Bb). The nuclear baskets had a diameter of 20-30 nm opening in the presence of calcium whereas the opening appeared closed in the absence of calcium. Furthermore, such open-close status was closely regulated as addition-depletion cycle of calcium in the stores.

\section{Conformational changes observed from both sides of NPC}

With the existing contradictions on the morphological changes in NPC, as to whether there is a shift towards the cytoplasmic side or the nuclear side of the NPC structure with calcium depletion from the lumen, a few others believe that there may be a graded displacement of the central mass towards both sides of NPC (Erickson et al., 2006). Dunn et al. found a shift of central granule up to $9 \mathrm{~nm}$ towards the cytoplasmic side and conformational changes on nuclear side at a decreased nuclear cisternal calcium concentration (Moore-Nichols et al., 2002). Activation of $\mathrm{IP}_{3}$ receptors (Stehno-Bittel et al., 1995a) and rynodine receptors (Erickson et al., 2004) induced calcium to be released from the cisternal spaces, and subsequently caused conformational changes in NPC with an apparent outward displacement of the central mass on both sides (Mooren et al., 2004). Several research 
groups also speculated that the FG Nups in the NPC might play a dominant role in the NPC conformational change. One possibility is that the FG Nups that line the nuclear pore could be induced to expand their spatial distributions towards both sides of the NPC (Stoffler et al., 1999). As a consequence, accessibility of the transiting cargo molecules to the $F G$ repeats and their binding affinities could be altered, which can in turn affect the nucleocytoplasmic transport mechanism. Fahrenkrog et al. studied spatial locations of two specific FG Nups (Nup153 and Nup214) locating far from the central pore region under different calcium concentrations, and found that these FG Nups constrains at the calcium-depletion condition. These studies suggest that calcium-induced alterations are not limited to the conformational change of the central framework of NPC, but also to the peripheral parts (Paulillo et al., 2006). The calcium pool in the NE lumen may modulate the structure of entire pore, and subsequently affect the NPC permeability. Indeed, as shown in the following section, an inherent structure-function relationship of NPC was observed in a calcium-dependent manner.

\section{CALCIUM-REGULATED FUNCTIONAL CHANGES IN NPC}

Structure determines functions. Whether and how the calcium-induced conformational changes in the NPC will affect the nucleocytoplasmic transport remains in dispute. In particular, over the years, different research groups presented two contrasting views to this argument. First, both the passive diffusion of small molecules and the facilitated translocation of larger molecules through the NPC could be inhibited when calcium is greatly depleted in calcium stores. Second, reversely, calcium-depletion has no effect on either passive diffusion or facilitated translocation.

\section{Nucleocytoplasmic transport is inhibited by depletion of calcium in the calcium stores}

Calcium-regulated passive diffusion of small molecules through the NPC was initially shown in isolated nuclei of Xenopus laevis oocyte by Stehno-Bittel et al. (1995b). By fluorescence microscopy, they observed that $10 \mathrm{kDa}$ dextran molecules conjugated with fluorophores were unable to enter the nucleus any longer after depletion of nuclear store calcium by $\operatorname{InsP}_{3} \mathrm{Rs}$ or calcium chelators. Whereas smaller molecules and ions, about the size of 500-dalton, diffused freely even after calcium store depletion. Another study by Greber et al. demonstrated that the alternation of calcium concentration in the lumen of NE also inhibited the passive diffusion of $10 \mathrm{kDa}$ dextran molecules (Greber and Gerace, 1995). In the experiments, dextran molecules were microinjected into the cytoplasm of ionophore- or thapsigargintreated NRK cells, and they found that the injected dextran molecules were just confined in the cytoplasm instead of entering the nucleus (Fig. 2Aa). A similar finding was reported by Perez-Terzic et al. (1997) in cardiomyocytes that calciumdepletion from the lumen led to an inhibition of fluorescently tagged dextran molecules $(3 \mathrm{kDa}$ and $10 \mathrm{kDa})$ through the NPC (Fig. 2Aa).

Besides the calcium-inhibited passive diffusion, Greber et al. also reported that alternation of the calcium concentration in the lumen of NE regulates active transport through NPCs (Greber and Gerace, 1995). In detail, treatment of cells with calcium depleting agents inhibited the nuclear import of both a fluorescent protein nucleoplasmin and an NLScontaining nuclear import ligand (Fig. 2Ac). Interestingly, the functional transport of large molecules was restored when the drugs depleting luminal calcium were removed and cells were incubated in a calcium-containing medium (Greber and Gerace, 1995). As an analogous to the studies on passive diffusion, Perez-Terzic et al. investigated active transport in cardiomyocytes, and found calcium-depletion from the lumen by calcium ion chelator or a calcium ion pump inhibitor or ionomycin caused the inhibition of nuclear import of histone $\mathrm{H} 1(\sim 21 \mathrm{kDa})$ (Fig. 2Ad). Restoration of the active transport was also observed when cells were re-incubated in calciumcontaining media (Perez-Terzic et al., 1999). Another interesting observation by Stehno-Bittel et al. was that ATP addition and depletion also regulates the facilitated transport of proteins (Perez-Terzic et al., 1999). Malviya et al. showed a link between $\mathrm{Ca}^{2+}$-ATPase phosphorylation and transport of intermediate size particles. In detail, a cAMP-dependent protein, kinase phosphorylated $\mathrm{Ca}^{2+}$-ATPase, resulted in calcium accumulation in the nuclear envelop, which in turn triggered the transport of molecules into the nucleus (Gensburger et al., 2003). All the above data indicate that NPC function is sensitive to the concentration of calcium within the NE. Altering luminal calcium concentration can regulate passive, intermediate, or receptor-mediated transport of molecules.

A connection between the calcium-regulated nuclear pore structure and the calcium-mediated active transport was set up via a Nup. An integral membrane protein gp210, a nucleoporin of $210 \mathrm{kDa}$ that functions to anchor NPCs, interacts with NPCs via its cytosolic C-terminus, whereas the bulk of the protein projects into the lumen of the NE, where it can sense calcium levels and thereby mediate changes in NPC structure (Greber et al., 1990). Experiments conducted with antibody specific for gp210 further confirmed the inhibition of both passive diffusion and signal-mediated transport into the nucleus by calcium-depletion in stores (Greber and Gerace, 1992).

\section{Nucleocytoplasmic transport is unaffected by depletion of calcium in the calcium stores}

Contrastingly, there are also some reports asserting that neither the passive diffusion of small molecules nor the signal-dependent facilitated translocation through NPC is affected by calcium concentrations in luminal calcium stores. 
A

a

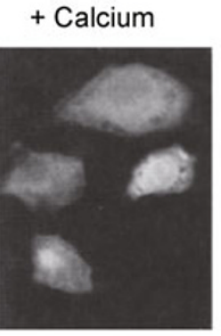

b

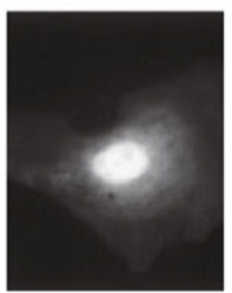

Passive diffusion is inhibited

c

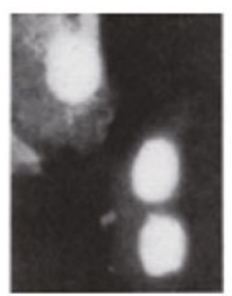

d

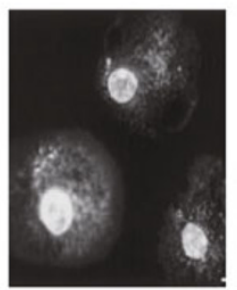

- Calcium
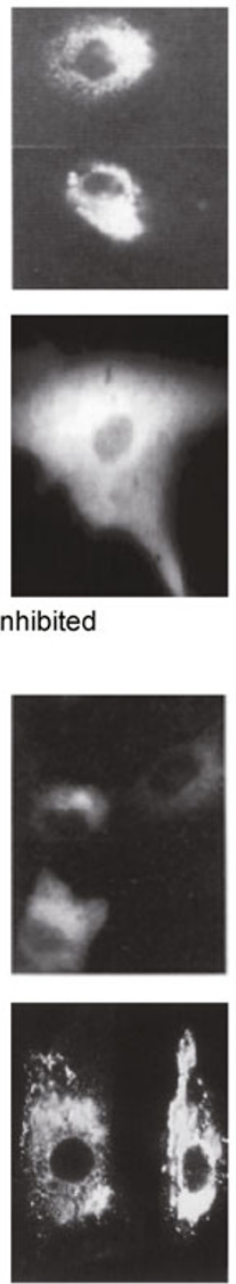

Facilitated transport is inhibited

B
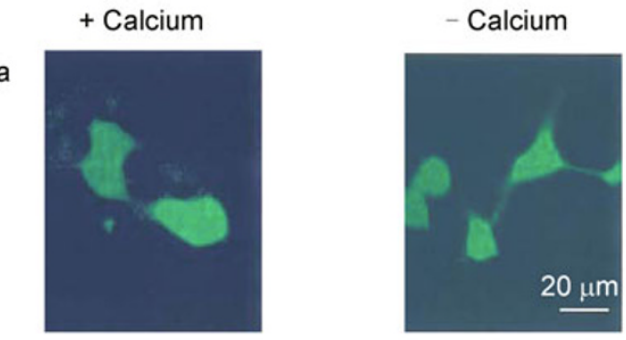

Passive diffusion is unaffected

b
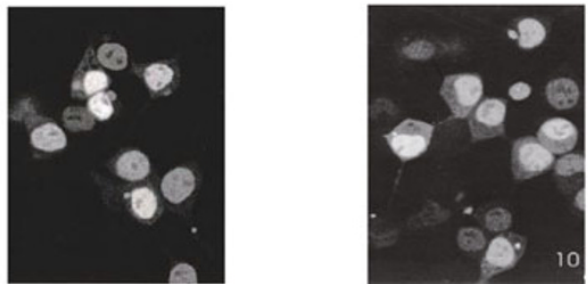

c
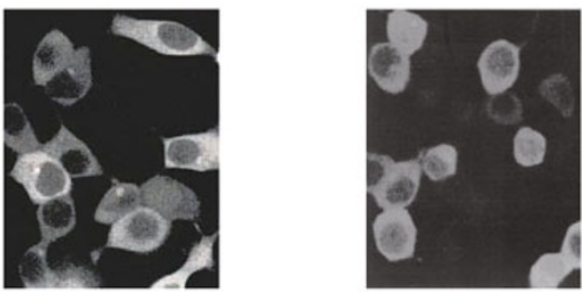

Facilitated transport is unaffected

Figure 2. Controversial data regarding calcium-mediated nucleocytoplasmic transport. (A) Nucleocytoplasmic transport of cargo molecules affected by calcium depletion from the stores. a. Distribution of fluorescent $10 \mathrm{kDa}$ dextran molecules in the cytoplasm and nucleus of NRK cells in the presence (+ Calcium) and absence (- Calcium) of calcium. Passive diffusion of dextran molecules is inhibited at calcium-depletion condition. Images adopted from the reference of (Greber and Gerace, 1995). b. Distribution of fluorescent $10 \mathrm{kDa}$ dextran molecules in the cytoplasm and nucleus of cardiomyocytes. Passive diffusion of molecules is also inhibited. Images adopted from the reference of (Perez-Terzic et al., 1999). c. Texas red-labeled nucleoplasmin is injected in the cytoplasm of NRK cells and its spatial distribution on either side of NE is measured in the presence and absence of calcium. Active transport of this cargo is inhibited in the absence of calcium. Images adopted from the reference of (Greber and Gerace, 1995). d. Distribution of fluorescein-labeled histone in the cytoplasm and nucleus of cardiomyocytes is measured. The active transport of histone molecules is also inhibited in the absence of calcium. Images adopted from the reference of (Perez-Terzic et al., 1999). (B) Some results demonstrated that nucleocytoplasmic transports of molecules were not regulated by the calcium depletion from the stores. a. No change was observed in passive transport of fluorescent $10 \mathrm{kDa}$ dextran in HM1 cells in the presence or absence of calcium. Images adopted from the reference of (Wei et al., 2003). b. Active nucleocytoplasmic transport of green fluorescent protein (GFP)-labeled glucocorticoid receptors in HM1 cells was monitored and there was no alternation between the presence and the absence of calcium. c. Facilitated translocation of GFP-MK2 in HM1 cells was not affected by either the presence or the absence of calcium. Images b and c are adopted from the reference of (Strübing and Clapham, 1999a). 
Oberleithenier et al. measured the passive diffusion rate of fluorophore-labeled $10 \mathrm{kDa}$ dextran molecules in an isolated Xenopus oocyte by confocal fluorescence microscopy and found that the diffusion rate was independent of varied calcium concentrations in calcium stores (Stehno-Bittel et al., 1995b). As shown in Fig. 2Ba, Strubing et al. confirmed the results (Wei et al., 2003). Moreover, the same research group used green fluorescent protein (GFP) tagged glucocorticoid receptor (GR-GFP), mitogen-activated protein (MAP) kinase activated protein kinase 2 (GFP-MK2), or SV-40 containing protein as substrates, to study the effect of calcium store depletion on active transport in HM1 cells, a human embryonic kidney cell line. They found that calcium-depletion in the stores by either carbachol, thapsigargin (Fig. 2Bb) or ionomycin had no effect on the import of these substrates (Fig. 2Bc). Altogether, they concluded that depletion of stored calcium is not directly linked to the inhibition of active protein transport through the NPC (Strübing and Clapham, 1999b).

\section{SINGLE-MOLECULE APPROACHES TO INVESTIGAE NUCLEOCYTOPLASMIC TRANSPORT}

Clearly, more strategies and studies are needed to clarify the observed conflicting roles calcium plays in regulating the function and structure of NPC. Moreover, a new method is urgently needed to associate calcium-induced conformational changes in NPC to the calcium-related functional alternations during the nucleocytoplasmic transport. Almost all previous investigations on calcium effect on nuclear pore permeability were carried out by the ensemble measurements of concentration ratio of transiting molecules between the cytoplasm and the nucleus (Greber and Gerace, 1995; Stehno-Bittel et al., 1995b; Lee et al., 1998; Wei et al., 2003; O'Brien et al., 2007). The use of ensemble measurements, which report changes that affect an averaged outcome, has been crucial in our understanding of nucleocytoplasmic transport. However, important information is lost by averaging signals, and determining molecular mechanism of nuclear transport necessitates studying individual transiting events. For example, the detailed transport information through the NPC, such as transport time, transport efficiency and spatial locations of transiting molecules, is inevitably missed due to a challenge of capturing transient movements of individual molecules within the sub-micrometer-sized NPC. Therefore, assessing this detailed dynamic information is beyond the capabilities of population methods and these parameters are crucial for a fundamental understanding of calcium-regulated transport mechanism through the NPC.

\section{Single-molecule fluorescence imaging and tracking}

An entirely new field of science, often referred to as singlemolecule biology, has emerged and developed rapidly in recent years. Single-molecule methods provide a truly innovative approach to investigate biological/biochemical/ biomedical problems by distinguishing, monitoring, tracking and controlling individual bio-molecules in vitro and in vivo. The classical ensemble methods, averaging the measured parameters over the entire bio-molecular populations, inevitably lost important information, such as transient intermediate status of molecules, a minority of spatially or temporally localized signals, and non-synchronized events. Singlemolecule methods provide the only available way to capture these import information. There are three main categories of single-molecule methods: mechanical, electrical and optical approach. Given its inherently noninvasive detection, fluorescence microscopy imaging emerges as the most proper method to observe specific components or processes in living cells, tissues, and whole organisms.

However, owing to diffraction, a wave of light cannot be focused to an arbitrarily small point. Conventional fluorescence microscopes therefore have long been thought to be incapable of resolving two objects closer together than about half the wavelength of light, with an imaging resolution of approximately $200 \mathrm{~nm}$ laterally and $600 \mathrm{~nm}$ axially. Recently, a number of fluorescence imaging techniques have pushed the boundaries of optical resolution below diffraction limit, as low as several nanometers (Hess et al., 1994; Betzig, 1995; Patterson and Lippincott-Schwartz, 2002; Thompson et al., 2002; Yildiz et al., 2003; Yildiz et al., 2004; Fernández-Suárez and Ting, 2008; Lippincott-Schwartz and Patterson, 2009). The fundamental principle is: the position of an isolated fluorescent emitter, although its image appears as a diffraction-limited spot, can be precisely determined by finding the centroid of its image. The precision of this localization process is determined by the background noise and the total number of photons collected from the single emitter. This concept has been used to track small particles with nanometer-scale accuracy. Recently it has been shown that, even when the emitter is a single fluorescent dye molecule, its position can be determined with a precision as high as $1 \mathrm{~nm}$ (Yildiz et al., 2003; Yildiz et al., 2004).

\section{Single-molecule fluorescence microscopy study of nucleocytoplasmic transport}

In fact, imaging and tracking individual transiting molecules through single NPCs will be an ideal approach to provide detailed kinetics and spatial properties in the NPC. To pursue the aim, deep penetration into the cell, fast imaging of single transiting molecules interacting with the NPC spanning over approximately $200 \mathrm{~nm}$ and high spatial-resolution localization of these single molecules are required. In the past years, epifluorescence microscopy approaches have been widely utilized as primary tools to elucidate the interaction time, the transport efficiency of individual transiting molecules through the NE and the relative spatial locations of transiting molecules from the middle plane of NE (Bustamante et al., 
2003; Yang et al., 2004; Kubitscheck et al., 2005; Zhuang, 2005; Yang and Musser, 2006a, b; Dange et al., 2008; Kahms et al., 2009; Cardarelli and Gratton, 2010). The capability and capacity of technical advance can determine the accuracies of above parameters. Conventional wide-field epifluorescence microscopy was employed to illuminate the entire NE and image single-molecule transport events through multiple NPCs on the NE. However, this technique suffers a low signal to noise $(\mathrm{S} / \mathrm{N})$ ratio for single-molecule imaging and tracking due to the significant noise of background fluorescence emitted from the out-of-focal-plane fluorescent molecules. To seek a higher $\mathrm{S} / \mathrm{N}$ ratio, a narrow-field epifluorescence microscopy was developed by introducing a micrometersized pinhole to confine the excitation light beam. A narrower excitation beam generates a smaller excitation volume in and out of the focal plane and greatly reduces the noise of background fluorescence (Fig. 3A). Such a modification can generate an approximately two-fold improvement in $\mathrm{S} / \mathrm{N}$ ratio (Yang and Musser, 2006b). The improved S/N ratio can enhance a better spatial localization of single fluorescent molecules. So far, the wide-field epifluorescence method can result in a spatial resolution of approximately $30 \mathrm{~nm}$ at $5 \mathrm{~ms}$ (Kubitscheck et al., 2005; Dange et al., 2008). However, in contrast, the narrow-field method can obtain a spatial resolution of about $15 \mathrm{~nm}$ at $2 \mathrm{~ms}$ (Yang et al., 2004; Yang and Musser, 2006a, b). The improved spatiotemporal resolution can enhance more accurate determination of transport kinetics on the NE. A recent technical advance by yielding a diffraction-limited off-axis illumination volume further enhances the spatiotemporal resolution to be $0.4 \mathrm{~ms}$ and 9 $\mathrm{nm}$ to map three-dimensional spatial distribution of transiting molecules in single NPCs (Ma and Yang, 2010).

These approaches provide great insights into nuclear transport mechanism. First, the transport of molecules through the NPC does not possess directional movements, rather, follows unbiased random diffusion (Yang et al., 2004). Second, the transport time of various molecules through the NPC is at millisecond level, which is much faster than hundreds of millisecond or seconds previously estimated by ensemble methods (Fig. 3B-D) (Yang et al., 2004; Kubitscheck et al., 2005; Yang and Musser, 2006a, b; Dange et al., 2008). Third, the observed abortive transport events reveal that not all transiting molecules can complete their transport as soon as they enter the NPC (Yang and Musser, 2006a). Forth, transport receptor-facilitated translocation of larger molecules separate their routes from passive diffusion of small molecules (Ma and Yang, 2010).

\section{Single-molecule fluorescence microscopy may provide new insights into calcium-mediated nucleocytoplasmic transport}

Single-molecule fluorescence microscopy approach may help resolve the ambiguity and confusion in both calcium-mediated nucleocytoplasmic transport and NPC conformational change. Undoubtedly, the nuclear pores are filled with natively unfolded FG-Nups, but the structure of this selective permeability barrier cannot be crystallized or imaged by AFM and EM. The newly developed super-resolution microscopy, single-point edge-excitation sub-diffraction (SPEED) microscopy may shed a light on this challenging puzzle (Ma and Yang, 2010). With a high spatiotemporal resolution of $400 \mu \mathrm{s}$ and $9 \mathrm{~nm}$, SPEED microscopy has been successfully applied to capture transient interactions between $\operatorname{Imp} \beta 1$ and the FG repeats in the natural NPC under physiological trafficking conditions. By measuring spatial locations of multiple transport receptors in the NPC, the spatial distribution of these locations could provide a final map of FG repeats in the nuclear pore. When the concentration of calcium ions is adjusted, the FG-repeat map can also be measured accordingly and the expected results would unambiguously indicate the changes. This could eventually solve the disputes of conformational changes induced by calcium on either side of NPC.

Till now, the calcium-mediated nucleocytoplasmic transport was monitored by measuring bulk transport rate between the cytoplasm and the nucleus. However, the transport rate is determined by multiple independent parameters: interaction frequency, transport time, and transport efficiency. The depletion of calcium may only alter one, two or all three in different measurements depending on instant experimental conditions. By bulk averaging methods, these parameters and their changes cannot be obtained. As demonstrated in the above, single-molecule approach picked up these numbers both in vitro and in vivo. With the knowledge of each parameter, we may fundamentally understand the mechanism of calcium-induced nucleocytoplasmic transport. First, transport time may suggest how the dimension, the viscosity of passageway for cargo molecule can be changed under different calcium conditions. Second, interaction frequency between cargo molecules and the NPC may indicate the spatial distribution of FG repeats and how they could be altered by calcium. Third, transport efficiency may reflect how deep the cargo molecules could penetrate in the NPC and how easily they could complete their passageways whatever import or export. Altogether, the information would provide innovative information and ultimately advance our understanding of calcium-mediated nucleocytoplasmic transport.

\section{SUMMARY AND PERSPECTIVE}

Over the past years, numerous studies on calcium-regulated structure and function of the NPC have greatly advanced our understanding of this unique mechanism. Clearly, many studies revealed that the structure of NPC can be altered at different calcium concentrations in the stores, but which part of the NPC topology changes is still debatable. As for the nucleocytoplasmic transport, the conflicting results that whether or not the transport is regulated by calcium remains 
A

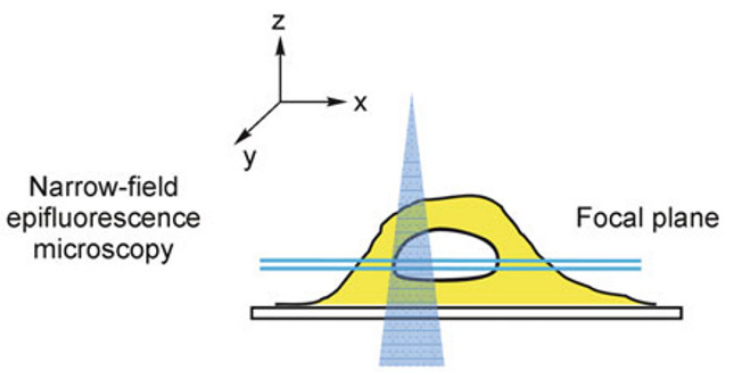

B
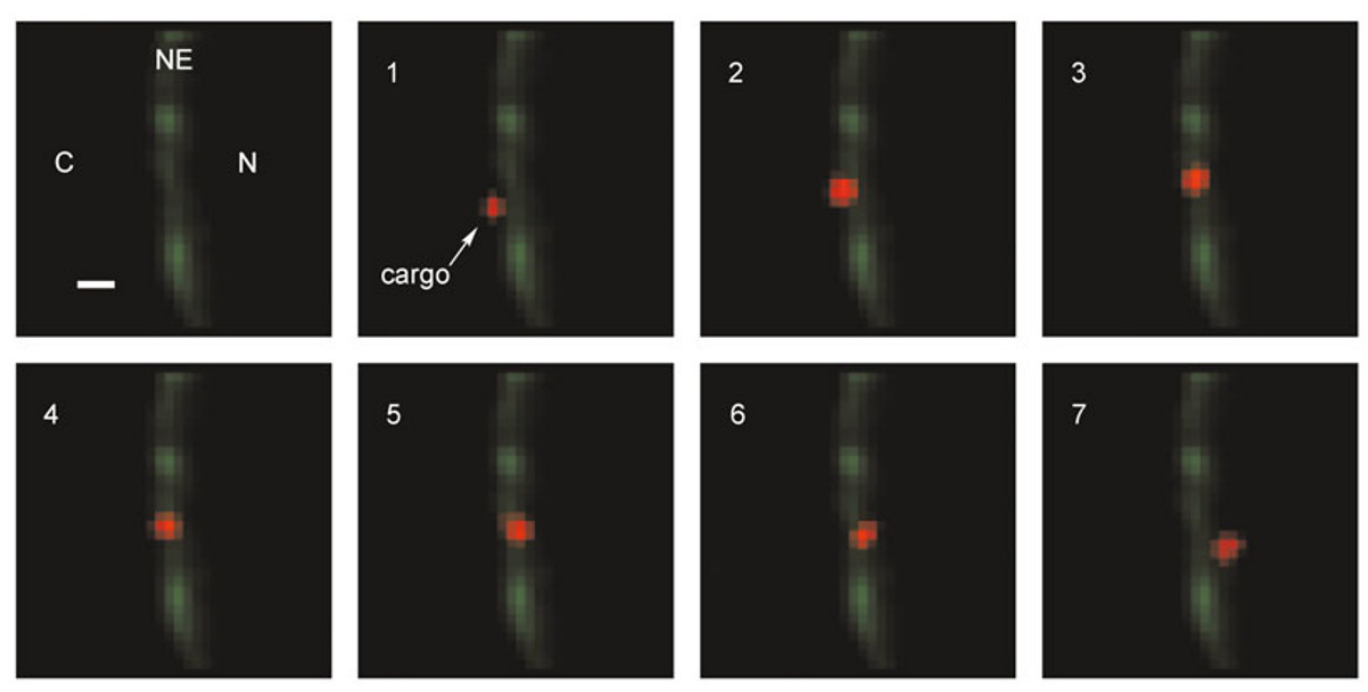

C

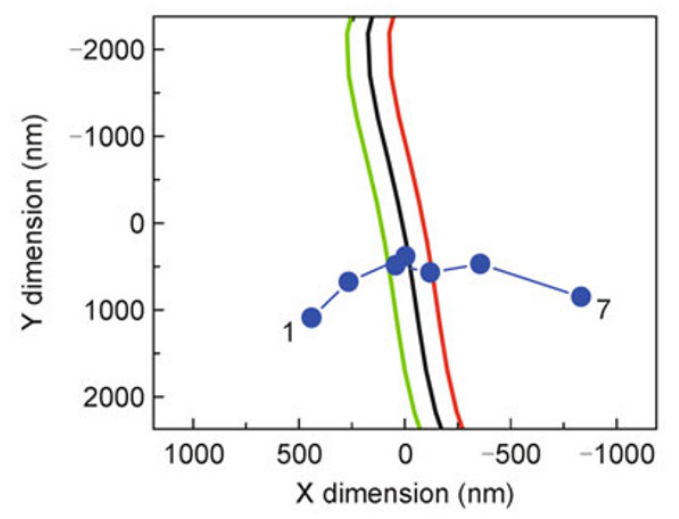

xz plane

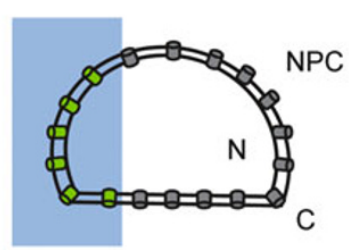

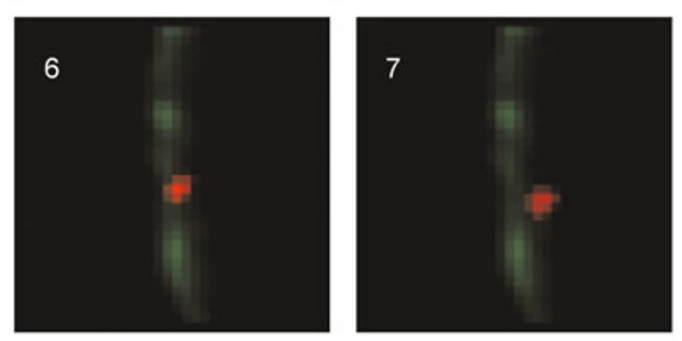

D

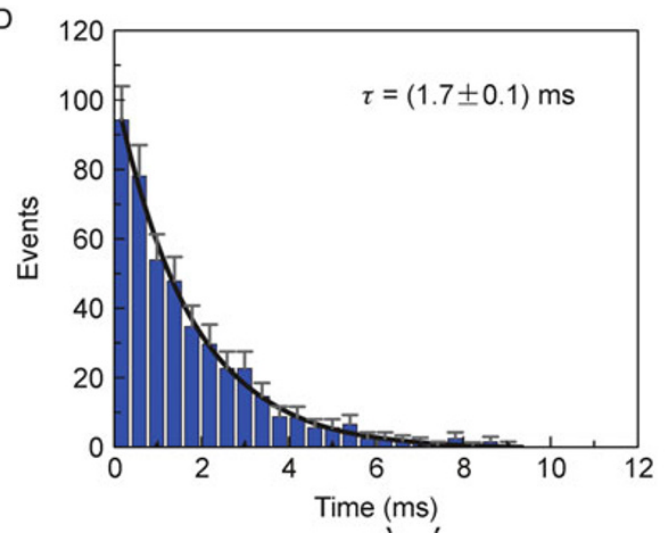

xy plane

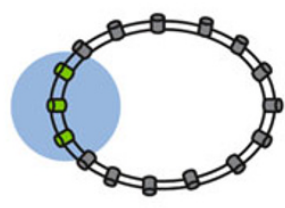

Figure 3. Single-molecule fluorescence microscopy imaging of nucleocytoplasmic transport. (A) The diagram demonstrates a single cell is imaged by the narrow-field epifluorescence microscopy. Because the illumination laser beam focused by objective was confined by a micrometer-sized pinhole, a small illumination volume was formed in the focal plane (double blue lines). As a result, partial NEs were imaged in both $x y$ and $x z$ planes. NPC, nuclear pore complex; C, the cytoplasm; N, the nucleus. (B) Select video frames demonstrating an import event in a permeabilized HeLa cell. A fluorescent cargo molecule (red dot, labeled with red fluorophores) started from the cytoplasm (C), interacted with the NPC (green line, POM121, a Nup of the NPC, conjugated with a green fluorescent protein), and entered the nucleus (N). Numbers correspond to frame. Each frame is $0.4 \mathrm{~ms}$. Scale bar: $1 \mu \mathrm{m}$. (C) Trajectories for the interaction event shown in (B) from frames 1 to 7 (blue dots). The black curve is the experimentally determined position of the NE from the fluorescent image of GFP-POM121. The green and red curves are for reference at $+100 \mathrm{~nm}$ and $-100 \mathrm{~nm}$ from the NE, respectively. (D) Histogram of interaction times for the cargo $(T=(1.7 \pm 0.1) \mathrm{ms}, \mathrm{N}=455)$. 
to be determined. The disputes may be due to variations in experimental procedures, technical limitations, types of cellular system and sample preparations. Undoubtedly, the lack of noninvasive detection of the dynamic changes in the native NPCs is still one of the biggest challenges for further understanding the precise role of calcium in NPC structural changes and nucleocytoplasmic transport.

With high spatiotemporal resolutions, the novel singlemolecule fluorescence microcopy has been proven an efficient approach to not only capture transient kinetics, but also determine spatial distribution of transiting molecules in the sub-micrometer-sized NPC. An expansion of the methods into the studies of calcium-regulated NPC could provide innovative information in both structure and function. Ultimately, a combination of multiple methodologies and a systematic analysis of the obtained data will continually advance our understanding of the calcium-regulation mechanism.

\section{ACKNOWLEDGEMENTS}

We thank the grant support from National Institutes of Health (GM094041-01) and the Research Capacity Enhancement Grant (Bowling Green State University).

\section{REFERENCES}

Bayliss, R., Littlewood, T., and Stewart, M. (2000). Structural basis for the interaction between FxFG nucleoporin repeats and importinbeta in nuclear trafficking. Cell 102, 99-108.

Beck, M., Förster, F., Ecke, M., Plitzko, J.M., Melchior, F., Gerisch, G., Baumeister, W., and Medalia, O. (2004). Nuclear pore complex structure and dynamics revealed by cryoelectron tomography. Science 306, 1387-1390.

Bednenko, J., Cingolani, G., and Gerace, L. (2003). Importin $\beta$ contains a $\mathrm{COOH}$-terminal nucleoporin binding region important for nuclear transport. J Cell Biol 162, 391-401.

Betzig, E. (1995). Proposed method for molecular optical imaging. Opt Lett 20, 237-239.

Bischoff, F.R., and Görlich, D. (1997). RanBP1 is crucial for the release of RanGTP from importin $\beta$-related nuclear transport factors. FEBS Lett 419, 249-254.

Bischoff, F.R., Klebe, C., Kretschmer, J., Wittinghofer, A., and Ponstingl, H. (1994). RanGAP1 induces GTPase activity of nuclear Ras-related Ran. Proc Natl Acad Sci U S A 91, 2587-2591.

Bootman, M.D., Fearnley, C., Smyrnias, I., MacDonald, F., and Roderick, H.L. (2009). An update on nuclear calcium signalling. J Cell Sci 122, 2337-2350.

Brohawn, S.G., Partridge, J.R., Whittle, J.R.R., and Schwartz, T.U. (2009). Nuclear pore complex has entered the atomic age. Structure 17, 1156-1168.

Bustamante, C., Bryant, Z., and Smith, S.B. (2003). Ten years of tension: single-molecule DNA mechanics. Nature 421, 423-427.

Cardarelli, F., and Gratton, E. (2010). In vivo imaging of singlemolecule translocation through nuclear pore complexes by pair correlation functions. PLoS One 5, e10475.

Carmen, P.T., Marisa, J., and David, E.C. (1997). Nuclear calcium and the regulation of the nuclear pore complex. BioEssays 19, 787-792 Cook, A., Bono, F., Jinek, M., and Conti, E. (2007). Structural biology of nucleocytoplasmic transport. Annu Rev Biochem 76, 647-671.

Corbett, A.H., and Silver, P.A. (1997). Nucleocytoplasmic transport of macromolecules. Microbiol Mol Biol Rev 61, 193-211.

Coutavas, E., Ren, M., Oppenheim, J.D., D'Eustachio, P., and Rush, M.G. (1993). Characterization of proteins that interact with the cellcycle regulatory protein Ran/TC4. Nature 366, 585-587.

Cronshaw, J.M., Krutchinsky, A.N., Zhang, W., Chait, B.T., and Matunis, M.J. (2002). Proteomic analysis of the mammalian nuclear pore complex. J Cell Biol 158, 915-927.

Dange, T., Grünwald, D., Grünwald, A., Peters, R., and Kubitscheck, U. (2008). Autonomy and robustness of translocation through the nuclear pore complex: a single-molecule study. J Cell Biol 183, $77-86$.

Danker, T. and Oberleithner, H. (2000). Nuclear pore function viewed with atomic force microscopy. Pflügers Arch 439, 671-681.

Denning, D.P., Patel, S.S., Uversky, V., Fink, A.L., and Rexach, M. (2003). Disorder in the nuclear pore complex: the FG repeat regions of nucleoporins are natively unfolded. Proc Natl Acad Sci U S A 100, 2450-2455.

Erickson, E.S., Mooren, O.L., Moore, D., Krogmeier, J.R., and Dunn, R. C. (2006). The role of nuclear envelope calcium in modifying nuclear pore complex structure. Can J Physiol Pharmacol 84, 309-318.

Erickson, E.S., Mooren, O.L., Moore-Nichols, D., and Dunn, R.C. (2004). Activation of ryanodine receptors in the nuclear envelope alters the conformation of the nuclear pore complex. Biophys Chem 112, 1-7.

Fahrenkrog, B., and Aebi, U. (2003). The nuclear pore complex: nucleocytoplasmic transport and beyond. Nat Rev Mol Cell Biol 4, 757-766.

Fernández-Suárez, M., and Ting, A.Y. (2008). Fluorescent probes for super-resolution imaging in living cells. Nat Rev Mol Cell Biol 9, 929-943.

Frey, N., McKinsey, T.A., and Olson, E.N. (2000). Decoding calcium signals involved in cardiac growth and function. Nat Med 6, 1221-1227.

Fried, H., and Kutay, U. (2003). Nucleocytoplasmic transport: taking an inventory. Cell Mol Life Sci 60, 1659-1688.

Gensburger, C., Freyermuth, S., Klein, C., and Malviya, A.N. (2003). In vivo nuclear $\mathrm{Ca}^{2+}$-ATPase phosphorylation triggers intermediate size molecular transport to the nucleus. Biochem Biophys Res Commun 303, 1225-1228.

Gerasimenko, J., Maruyama, Y., Tepikin, A., Petersen, O.H., and Gerasimenko, O. (2003). Calcium signalling in and around the nuclear envelope. Biochem Soc Trans 31, 76-78.

Greber, U.F., and Gerace, L. (1992). Nuclear protein import is inhibited by an antibody to a lumenal epitope of a nuclear pore complex glycoprotein. J Cell Biol 116, 15-30.

Greber, U.F., and Gerace, L. (1995). Depletion of calcium from the lumen of endoplasmic reticulum reversibly inhibits passive diffusion and signal-mediated transport into the nucleus. J Cell Biol 128, 5-14.

Greber, U.F., Senior, A., and Gerace, L. (1990). A major glycoprotein of the nuclear pore complex is a membrane-spanning polypeptide with a large lumenal domain and a small cytoplasmic tail. EMBO J 9, 1495-1502.

Hess, H.F., Betzig, E., Harris, T.D., Pfeiffer, L.N., and West, K.W. 
(1994). Near-field spectroscopy of the quantum constituents of a luminescent system. Science 264, 1740-1745.

Isgro, T.A., and Schulten, K. (2005). Binding dynamics of isolated nucleoporin repeat regions to importin-beta. Structure 13 1869-1879.

Izaurralde, E., Kutay, U., von Kobbe, C., Mattaj, I.W., and Görlich, D. (1997). The asymmetric distribution of the constituents of the Ran system is essential for transport into and out of the nucleus. EMBO J 16, 6535-6547.

Jäggi, R.D., Franco-Obregón, A., Mühlhäusser, P., Thomas, F., Kutay, U., and Ensslin, K. (2003). Modulation of nuclear pore topology by transport modifiers. Biophys J 84, 665-670.

Kahms, M., Lehrich, P., Hüve, J., Sanetra, N., and Peters, R. (2009). Binding site distribution of nuclear transport receptors and transport complexes in single nuclear pore complexes. Traffic 10, $1228-1242$.

Kass, G.E., and Orrenius, S. (1999). Calcium signaling and cytotoxicity. Environ Health Perspect 107, 25-35.

Kau, T.R., Way, J.C., and Silver, P.A.(2004). Nuclear transport and cancer: from mechanism to intervention. Nat Rev Cancer 4, 106-117.

Kramer, A., Ludwig, Y., Shahin, V., and Oberleithner, H. (2007). A pathway separate from the central channel through the nuclear pore complex for inorganic ions and small macromolecules. J Biol Chem 282, 31437-31443.

Kubitscheck, U., Grünwald, D., Hoekstra, A., Rohleder, D., Kues, T., Siebrasse, J.P., and Peters, R. (2005). Nuclear transport of single molecules: dwell times at the nuclear pore complex. J Cell Biol 168, 233-243.

Lee, M.A., Dunn, R.C., Clapham, D.E., and Stehno-Bittel, L. (1998). Calcium regulation of nuclear pore permeability. Cell Calcium 23, 91-101.

Lee, S.J., Matsuura, Y., Liu, S.M., and Stewart, M. (2005). Structural basis for nuclear import complex dissociation by RanGTP. Nature 435, 693-696.

Lim, R., Aebi, U., and Fahrenkrog, B. (2008). Towards reconciling structure and function in the nuclear pore complex. Histoche Cell Biol 129, 105-116.

Lim, R.Y.H., Fahrenkrog, B., Köser, J., Schwarz-Herion, K., Deng, J., and Aebi, U. (2007). Nanomechanical Basis of Selective Gating by the Nuclear Pore Complex. Science 318, 640-643.

Lippincott-Schwartz, J., and Patterson, G.H. (2009). Photoactivatable fluorescent proteins for diffraction-limited and super-resolution imaging. Trends Cell Biol 19, 555-565.

Liu, S.M., and Stewart, M. (2005). Structural basis for the high-affinity binding of nucleoporin Nup1p to the Saccharomyces cerevisiae importin-beta homologue, Kap95p. J Mol Biol 349, 515-525.

Lyman, S.K., Guan, T., Bednenko, J., Wodrich, H., and Gerace, L. (2002). Influence of cargo size on Ran and energy requirements for nuclear protein import. J Cell Biol 159, 55-67.

Ma, J., and Yang, W. (2010). Three-dimensional distribution of transient interactions in the nuclear pore complex obtained from single-molecule snapshots. Proc Natl Acad Sci U S A 107, 7305-7310.

Malviya, A.N., and Klein, C. (2006). Mechanism regulating nuclear calcium signaling. Can J Physiol Pharmacol 84, 403-422.

Mattson, M.P., LaFerla, F.M., Chan, S.L., Leissring, M.A., Shepel, P. N., and Geiger, J.D. (2000). Calcium signaling in the ER: its role in neuronal plasticity and neurodegenerative disorders. Trends Neurosci 23, 222-229.

Miao, L., and Schulten, K. (2009). Transport-related structures and processes of the nuclear pore complex studied through molecular dynamics. Structure 17, 449-459.

Moore, M.S., and Blobel, G. (1993). The GTP-binding protein Ran/ TC4 is required for protein import into the nucleus. Nature 365 , 661-663.

Moore-Nichols, D., Arnott, A., and Dunn, R.C. (2002). Regulation of nuclear pore complex conformation by $\mathrm{IP}(3)$ receptor activation. Biophys J 83, 1421-1428.

Mooren, O.L., Erickson, E.S., Moore-Nichols, D., and Dunn, R.C. (2004). Nuclear side conformational changes in the nuclear pore complex following calcium release from the nuclear membrane. Phys Biol 1, 125-134.

O'Brien, E.M., Gomes, D.A., Sehgal, S., and Nathanson, M.H. (2007). Hormonal Regulation of Nuclear Permeability. J Biol Chem 282, 4210-4217.

Patel, S.S., Belmont, B.J., Sante, J.M., and Rexach, M.F. (2007). Natively unfolded nucleoporins gate protein diffusion across the nuclear pore complex. Cell 129, 83-96.

Patterson, G.H., and Lippincott-Schwartz, J. (2002). A photoactivatable GFP for selective photolabeling of proteins and cells. Science 297, 1873-1877.

Paulillo, S.M., Powers, M.A., Ullman, K.S., and Fahrenkrog, B. (2006). Changes in nucleoporin domain topology in response to chemical effectors. J Mol Biol 363, 39-50.

Perez-Terzic, C., Gacy, A.M., Bortolon, R., Dzeja, P.P., Puceat, M., Jaconi, M., Prendergast, F.G., and Terzic, A. (1999). Structural plasticity of the cardiac nuclear pore complex in response to regulators of nuclear import. Circ Res 84, 1292-1301.

Perez-Terzic, C., Jaconi, M., and Clapham, D.E. (1997). Nuclear calcium and the regulation of the nuclear pore complex. BioEssays 19, 787-792

Perez-Terzic, C., Pyle, J., Jaconi, M., Stehno-Bittel, L., and Clapham, D.E. (1996). Conformational states of the nuclear pore complex induced by depletion of nuclear $\mathrm{Ca}^{2+}$ stores. Science 273 , 1875-1877.

Peters, R. (2009). Translocation through the nuclear pore: Kaps pave the way. Bioessays 31, 466- 477.

Rakowska, A., Danker, T., Schneider, S.W., and Oberleithner, H. (1998). ATP-Induced shape change of nuclear pores visualized with the atomic force microscope. J Membr Biol 163, 129-136.

Rexach, M., and Blobel, G. (1995). Protein import into nuclei: association and dissociation reactions involving transport substrate, transport factors, and nucleoporins. Cell 83, 683-692.

Rizzuto, R., and Pozzan, T. (2003). When calcium goes wrong: genetic alterations of a ubiquitous signaling route. Nat Genet 34 , 135-141.

Rout, M.P., and Aitchison, J.D. (2001). The nuclear pore complex as a transport machine. J Biol Chem 276, 16593-16596.

Rout, M.P., Aitchison, J.D., Magnasco, M.O., and Chait, B.T. (2003). Virtual gating and nuclear transport: the hole picture. Trends Cell Biol 13, 622-628.

Rout, M.P., Aitchison, J.D., Suprapto, A., Hjertaas, K., Zhao, Y., and Chait, B.T. (2000). The yeast nuclear pore complex: composition, architecture, and transport mechanism. J Cell Biol 148, 635-651.

Rout, M.P., and Blobel, G. (1993). Isolation of the yeast nuclear pore 
complex. J Cell Biol 123, 771-783.

Rout, M.P., and Wente, S.R. (1994). Pores for thought: nuclear pore complex proteins. Trends Cell Biol 4, 357-365.

Stehno-Bittel, L., Lückhoff, A., and Clapham, D.E. (1995a). Calcium release from the nucleus by InsP3 receptor channels. Neuron 14, 163-167.

Stehno-Bittel, L., Perez-Terzic, C., and Clapham, D.E. (1995b). Diffusion across the nuclear envelope inhibited by depletion of the nuclear Ca2 + store. Science 270, 1835-1838.

Stewart, M. (2007). Molecular mechanism of the nuclear protein import cycle. Nat Rev Mol Cell Biol 8, 195-208.

Stoffler, D., Feja, B., Fahrenkrog, B., Walz, J., Typke, D., and Aebi, U. (2003). Cryo-electron tomography provides novel insights into nuclear pore architecture: implications for nucleocytoplasmic transport. J Mol Biol 328, 119-130.

Stoffler, D., Goldie, K.N., Feja, B., and Aebi, U. (1999). Calciummediated structural changes of native nuclear pore complexes monitored by time-lapse atomic force microscopy. J Mol Biol 287, 741-752.

Stoffler, D., Schwarz-Herion, K., Aebi, U., and Fahrenkrog, B. (2006). Getting across the nuclear pore complex: new insights into nucleocytoplasmic transport. Can J Physiol Pharmacol 84, 499-507.

Strawn, L.A., Shen, T., Shulga, N., Goldfarb, D.S., and Wente, S.R. (2004). Minimal nuclear pore complexes define $F G$ repeat domains essential for transport. Nat Cell Biol 6, 197-206.

Strübing, C., and Clapham, D.E. (1999). Active nuclear import and export is independent of lumenal $\mathrm{Ca}^{2+}$ stores in intact mammalian cells. J Gen Physiol 113, 239-248.

Suntharalingam, M., and Wente, S.R. (2003). Peering through the pore: nuclear pore complex structure, assembly, and function. Dev Cell 4, 775-789.

Thompson, R.E., Larson, D.R., and Webb, W.W. (2002). Precise nanometer localization analysis for individual fluorescent probes.
Biophys J 82, 2775-2783.

Walther, T.C., Pickersgill, H.S., Cordes, V.C., Goldberg, M.W., Allen, T.D., Mattaj, I.W., and Fornerod, M. (2002). The cytoplasmic filaments of the nuclear pore complex are dispensable for selective nuclear protein import. J Cell Biol 158, 63-77.

Wang, H., and Clapham, D.E. (1999). Conformational changes of the in situ nuclear pore complex. Biophys J 77, 241-247.

Wei, X., Henke, V.G., Strübing, C., Brown, E.B., and Clapham, D.E. (2003). Real-time imaging of nuclear permeation by EGFP in single intact cells. Biophys J 84, 1317-1327.

Weis, K. (2003). Regulating access to the genome: nucleocytoplasmic transport throughout the cell cycle. Cell 112, 441-451.

Wozniak, R.W., Rout, M.P., and Aitchison, J.D. (1998). Karyopherins and kissing cousins. Trends Cell Biol 8, 184-188.

Yang, W., Gelles, J., and Musser, S.M. (2004). Imaging of singlemolecule translocation through nuclear pore complexes. Proc Natl Acad Sci U S A 101, 12887-12892.

Yang, W., and Musser, S.M. (2006a). Nuclear import time and transport efficiency depend on importin $\beta$ concentration. J Cell Biol 174, 951-961.

Yang, W., and Musser, S.M. (2006b). Visualizing single molecules interacting with nuclear pore complexes by narrow-field epifluorescence microscopy. Methods 39, 316-328.

Yildiz, A., Forkey, J.N., McKinney, S.A., Ha, T., Goldman, Y.E., and Selvin, P.R. (2003). Myosin V walks hand-over-hand: single fluorophore imaging with $1.5-\mathrm{nm}$ localization. Science 300 , 2061-2065.

Yildiz, A., Tomishige, M., Vale, R.D., and Selvin, P.R. (2004). Kinesin walks hand-over-hand. Science 303, 676-678.

Yokoyama, N., Hayashi, N., Seki, T., Panté, N., Ohba, T., Nishii, K., Kuma, K., Hayashida, T., Miyata, T., Aebi, U., et al. (1995). A giant nucleopore protein that binds Ran/TC4. Nature 376, 184-188.

Zhuang, X. (2005). Single-molecule RNA science. Annu Rev Biophys Biomol Struct 34, 399-414. 